\title{
Suppression of cell growth and invasion by miR-205 in breast cancer
}

\author{
Hailong $\mathrm{Wu}^{1}$, Shoumin $\mathrm{Zhu}{ }^{1}$, Yin-Yuan $\mathrm{Mo}^{1}$ \\ ${ }^{I}$ Department of Medical Microbiology, Immunology and Cell Biology, Southern Illinois University School of Medicine, 825 N. Rut- \\ ledge, PO Box 19626, Springfield, IL 62794, USA
}

MicroRNAs (miRNAs) are endogenous, small, non-coding RNAs, which are capable of silencing gene expression at the post-transcriptional level. In this study, we report that miR-205 is significantly underexpressed in breast tumor compared to the matched normal breast tissue. Similarly, breast cancer cell lines, including MCF-7 and MDA-MB231, express a lower level miR-205 than the non-malignant MCF-10A cells. Of interest, ectopic expression of miR-205 significantly inhibits cell proliferation and anchorage independent growth, as well as cell invasion. Furthermore, miR205 was shown to suppress lung metastasis in an animal model. Finally, western blot combined with the luciferase reporter assays demonstrate that ErbB3 and vascular endothelial growth factor A (VEGF-A) are direct targets for $m i R-205$, and this miR-205-mediated suppression is likely through the direct interaction with the putative miR-205 binding site in the 3'-untranslated region (3'-UTR) of ErbB3 and VEGF-A. Together, these results suggest that miR205 is a tumor suppressor in breast cancer.

Keywords: breast cancer, cell growth, ErbB3, miRNA, miR-205, post-transcriptional regulation, VEGF-A

Cell Research (2009) 19:439-448. doi: 10.1038/cr.2009.18; published online 24 February 2009

\section{Introduction}

MicroRNAs (miRNAs) are endogenously processed non-coding RNAs, which regulate gene expression by blocking the translation or decreasing the stability of mRNAs [1-3]. They are initially transcribed by RNA polymerase II into larger transcripts called pri-miRNAs [4-6]. In the nucleus, the RNase III Drosha interacting with DGCR8 cleaves pri-miRNAs to form pre-miRNAs [7]. The pre-miRNAs are then exported by exportin-5 to the cytoplasm, where Dicer further processes them into mature double-stranded miRNAs that are $\sim 22 \mathrm{nt}$ in length $[1,8]$. Finally, one strand of mature miRNA duplex is incorporated into RNA-induced silencing complex and guides the complex to target mRNAs, leading to post-

Correspondence: Yin-Yuan Mo

Tel: +1-217-545-8508

E-mail: ymo@siumed.edu

Abbreviations: EMT (epithelial to mesenchymal transition); miRNA (microRNA); RT (reverse transcription); UTR (untranslated region)

Received 24 June 2008; revised 8 September 2008; accepted 26 September 2008; published online 24 February 2009 transcriptional repression [1-3]. It is generally believed that base pairing between seed sequences (nucleotides 2-7) of miRNAs and target mRNAs is critical for target recognition, although there are reports that the seed sequence base pairing may not be sufficient to induce miRNA-mediated translation repression, suggesting the possible involvement of other components in miRNA: mRNA interactions [9-11].

To date, over 600 human miRNAs have been reported, and more than 1000 predicted miRNA genes remain to be experimentally confirmed $[12,13]$. As a new class of regulatory molecules, miRNAs exert diverse functions in a broad range of biological events, including cell growth, apoptosis and differentiation, as well as viral infection [1, 14-16]. Based on computer-aided predictions, miRNAs are estimated to target about one-third of the human genes [17]; so it is not surprising that miRNAs could play a key role in human malignancy. The role of miRNAs in human cancers was initially demonstrated in chronic lymphocytic leukemia (CLL), where a loss of $m i R-15 a$ and $m i R-16-1$ due to the $13 \mathrm{q} 14$ deletion was found in over half of CLL cases [18]. Since then, the deregulation of miRNA expression has been demonstrated in other types of cancers [19-21]. Of interest, many 
miRNAs are located at fragile sites or cancer-associated regions, which may explain why there is a correlation between tumorigenesis and aberrant expression of certain miRNAs [22]. Although a large number of miRNAs have been identified to date, the role for many of them in tumorigenesis and the underlying mechanisms remain to be determined.

$m i R-205$ is a highly conserved miRNA among different species (http://microrna. sanger.ac.uk/cgi-bin/ sequences/query.pl?terms $=$ mir-205). $m i R-205$ was first predicted by computational methods based on the conservation with mouse and Fugu rubripes sequences [23], and its expression was later validated in zebrafish and humans $[24,25]$. Human miR-205 is located in the second intron of LOC642587 locus in chromosome 1. Tissue-specific expression of $m i R-205$ has been previously reported. In zebrafish, $m i R-205$ is expressed in epidermis [26], and in humans, $m i R-205$ is detected in breast, prostate and thymus, suggesting that miR-205 may play a role in the development of these organs [27].

In this study, we report that $m i R-205$ is downregulated in breast tumor tissues as well as breast cancer cell lines; in contrast, $m i R-205$ is highly expressed in normal breast tissues and non-malignant breast epithelial cell line, MCF-10A. These results are in good agreement with the previous report [21]. Importantly, ectopic expression of $m i R-205$ significantly reduces cellular proliferation, clonogenic survival and anchorage-independent growth in breast cancer MCF-7 cells. In addition, $m i R-205$ is able to suppress invasion and metastasis of breast cancer MDA-MB-231 cells. We further demonstrated that $m i R$ 205 could specifically suppress expression of ErbB3 and
VEGF-A by directly interacting with the putative $m i R$ 205 binding site at the 3 '-UTR (3'-untranslated region).

\section{Results}

miR-205 is downregulated in breast tumor tissues and breast cancer cell lines

Accumulating evidence indicates that miRNAs can function as oncogenes or tumor suppressors by targeting corresponding tumor suppressor genes or oncogenes [28]. To determine the role of miRNAs in breast cancer, we profiled miRNA expression in matched breast tumor specimens by using miRNA TaqMan real-time PCR. We previously showed that the oncogenic $m i R-21$ was more highly expressed in breast tumors than in the matched normal breast tissues [29]. In contrast, we detected substantial downregulation of $m i R-205$ in breast cancer specimens based on our initial profiling. Therefore, we used single $m i R-205$ primer set and probe to confirm the preliminary finding. From a total of 19 pairs of matched breast tumor tissue specimens, the average $C_{\mathrm{T}}$ (threshold cycle) value for the normal tissue was $\sim 25$, whereas the $C_{\mathrm{T}}$ value for tumors was $\sim 28$ (Figure 1A). After conversion [30], $m i R-205$ expression level, on average, was downregulated in breast tumor tissues by $\sim 80 \%$ (Figure 1B) compared to the matched normal tissue. We also examined miR-205 expression in nine pairs of matched colon cancer specimens, but found no significant difference in $m i R-205$ expression level between tumors and the matched normal colon tissues (data not shown), suggesting that downregulation of $m i R-205$ may be specific to breast cancer. We then determined miR-205 expression
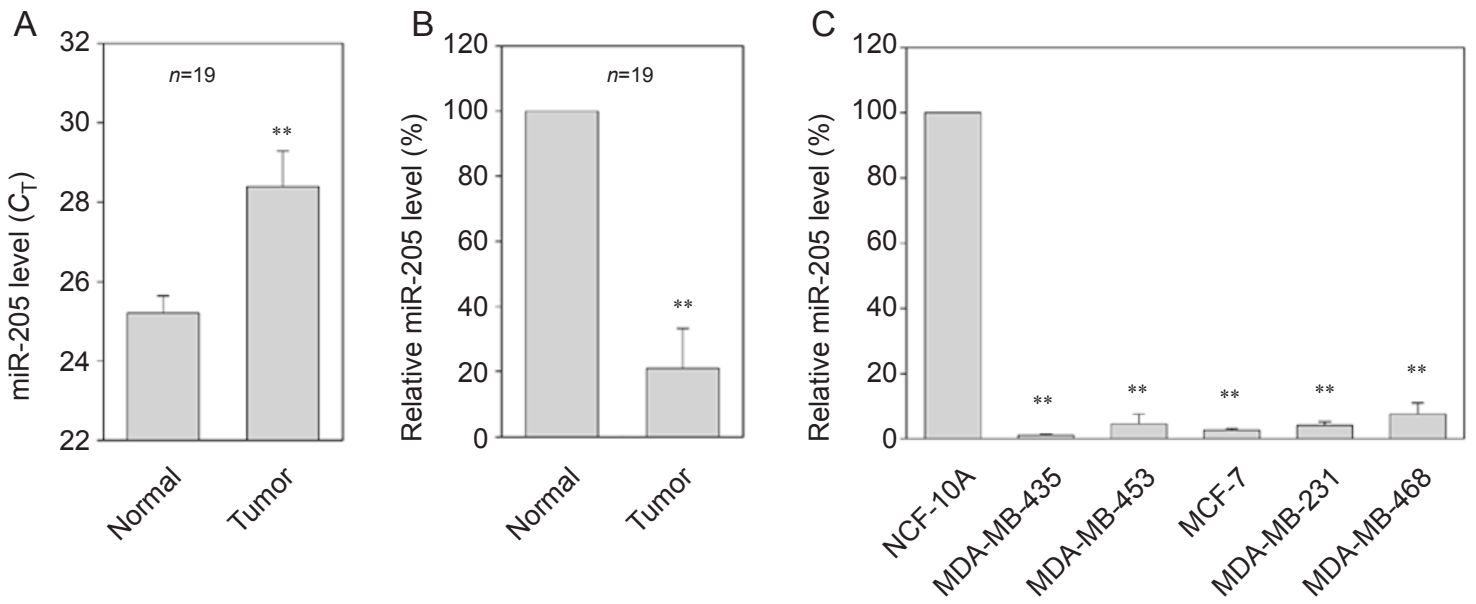

Figure 1 Expression of miR-205 in breast cancer specimens and breast cancer cell lines. miR-205 expression was determined by TaqMan real-time PCR method as described in Materials and Methods. Relative $C_{\mathrm{T}}$ value $(\mathrm{A})$ or relative expression level of miR-205 (B) in the matched breast tumors and normal breast tissue. (C) Relative expression of miR-205 in breast cancer cell lines compared to non-malignant MCF-10A cells from three separate experiments (mean $\pm \mathrm{SE})$. ** $P<0.01$. 

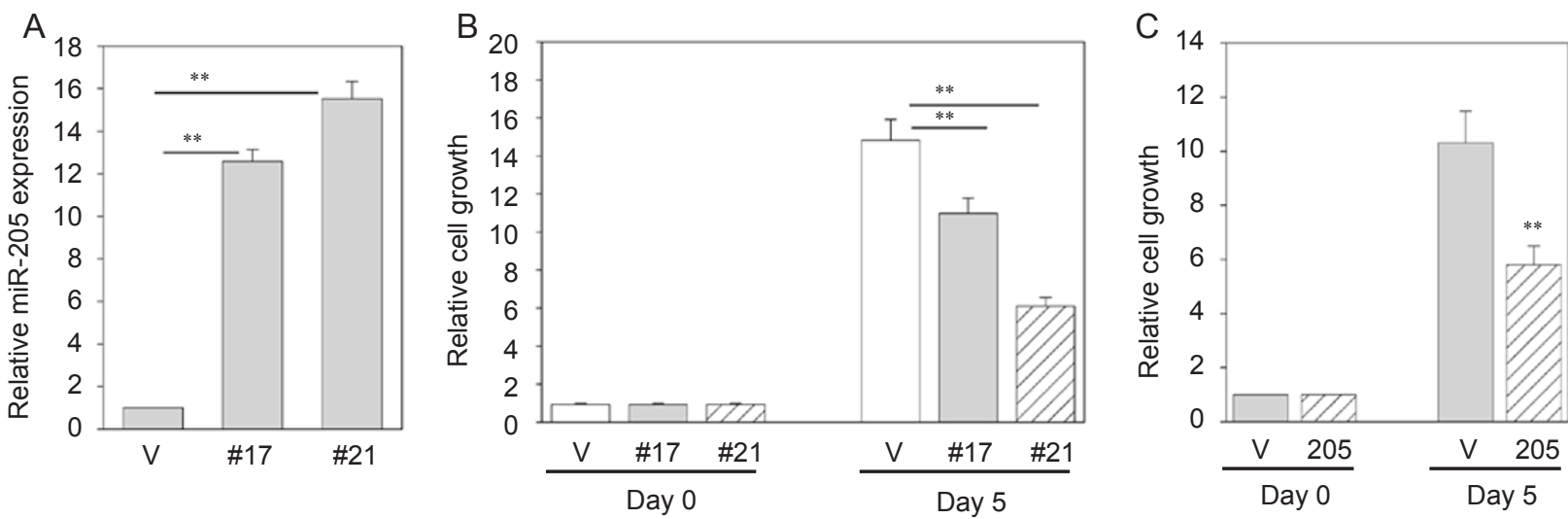

Figure 2 Suppression of cell growth by miR-205. (A) Relative expression of miR-205 in stable clones \#17 (miR-205 \#17) and \#21 (miR-205 \#21), as detected by TaqMan real-time PCR. MTT assays reveal reduced cell growth for these two stable clones compared to vector control $(\mathrm{V})(\mathrm{B})$, as well as for the transiently transfected MCF-7 cells compared to vector control (C). Values are means of three separate experiments \pm SE. $* * P<0.01$.

in various breast cancer cell lines along with the nonmalignant breast epithelial cell line MCF-10A. As shown in Figure 1C, breast cancer cell lines expressed lower levels of $m i R-205$ as compared with MCF-10A cells. These results suggest that $m i R-205$ may function as a tumor suppressor in breast cancer.

\section{Growth inhibition of MCF-7 cells by miR-205}

To test this hypothesis, we performed proliferation assays in MCF-7 cells with $m i R-205$ overexpression. MCF-7 cells were transfected with either $m i R-205$ expression vector or control vector. Two stable clones, $\# 17$ and \#21, were selected out based on the high level of exogenous $m i R-205$ level (Figure 2A) compared to a pooled vector control (over 20 clones). MTT assays indicated that cell growth for both miR-205-expressing clones was slower than vector control. For example, clones $m i R-205 \# 17$ and $m i R-205 \# 21$ grew at $60 \%$ and $30 \%$ of the vector control, respectively (Figure $2 \mathrm{~B}$ ), which appears to be negatively correlated with the exogenous miR-205 level (Figure 2A). We also tested transiently transfected MCF-7 cells. The result was very similar to that of stable clones (Figure 2C).

miR-205 suppresses clonogenic survival and anchorageindependent growth

To assess whether miR-205 inhibits clonogenic survival, colony formation assay was performed in MCF-7 stable clones. Compared to control vector, the number of colonies for miR-205 \#17 and miR-205 \#21 was decreased to about $63 \%$ and $56 \%$, respectively (Figure $3 \mathrm{~A}$ and $3 \mathrm{~B}$ ). Since anchorage-independent growth is strongly correlated with tumorigenicity [31], we then determined whether miR-205 inhibits anchorage-independent growth. Thus, the same MCF-7-stable transfectants were plated in soft agar and incubated for 2 weeks before counting the number of colonies. The ability to form colonies in soft agar was drastically decreased in stable transfectants with miR-205 expression compared to vector control (Figure 3C-3E).

miR-205 suppresses invasiveness in MDA-MB-231 cells

Based on miRNA profiling data, breast cancers without vascular invasion show high $m i R-205$ expression compared to breast cancers with vascular invasion [21]. Furthermore, a recent report indicates that miR205 is dramatically downregulated in cells undergoing epithelial to mesenchymal transition (EMT) [32]. These results suggest that $m i R-205$ may also play a role in cell invasion. To test this hypothesis, we chose a metastatic breast cancer cell line MDA-MB-231 because these cells also expressed a low level of $m i R-205$. Matrigel chamber assays indicated that the invasion ability of MDA-MB231 cells was substantially reduced by $m i R-205$ (Figure $4 \mathrm{~A}$ and $4 \mathrm{~B}$ ). Since invasion is one of the key steps in metastasis [33], suppression of invasion by $m i R-205$ in MDA-MB-231 cells suggests that $m i R-205$ may also affect breast cancer metastasis. In vivo metastasis assays supported this notion (Figure 4C and 4D). For example, while average number of lung nodules in the vector control cells was about 24, this number was only about 2 in the miR-205-expressing cells.

miR-205 directly targets ErbB3 and VEGF-A expression

To understand the molecular mechanisms by which $m i R-205$ inhibits tumor cell growth and cell invasion, 

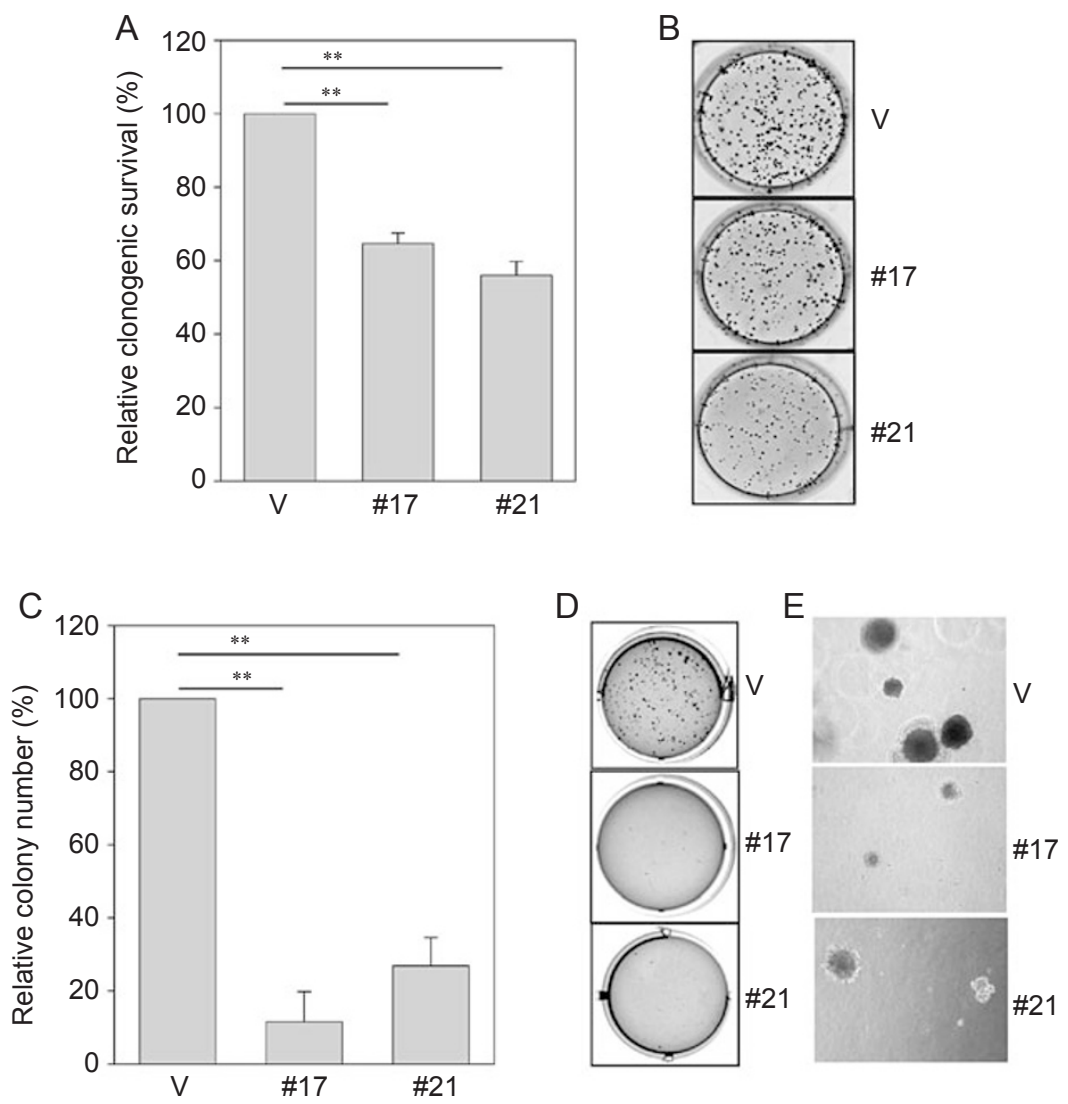

Figure 3 Suppression of clonogenic survival and anchorage-independent growth by miR-205. Clonogenic assay (A and B) and soft agar assay (C-E) were performed as described in Materials and Methods. (B) Representative wells of the colonies. (D) Representative fields of colonies in soft agar, and (E) closeups of the formed colonies. Note that the colonies are larger in vector control than miR-205 \#17 or \#21. Values in (A and C) are means of three separate experiments \pm SE. V, vector; \#17, miR-205\#17; \#21, miR-205 \#21; **P $<0.01$.

we searched for putative $m i R-205$ targets as predicted by the commonly cited programs such as TargetScan4 [11], miRBase Target5 (http://microrna.sanger.ac.uk/targets/ v5/), PicTar [34] and miRanda (http://www.microrna. org) [35]. This search identified several genes that are likely associated with tumorigenicity, including K-RAS, ESRRG (estrogen-related receptor gamma), Bcl-2, eIF4E, ErbB3 and VEGF-A. Therefore, we constructed luciferase reporters carrying the $3^{\prime}$-UTR with the putative $m i R-205$ binding sites for each of those genes. Luciferase assays indicated that the 3'-UTRs of both ErbB3 and VEGF-A gave rise to a significant reduction in the luciferase activity. The importance of ErbB3 is highlighted by the fact that ErbB3 is frequently overexpressed in breast cancer and ErbB2/ErbB3 heterodimer is the most potent oncogenic complex [36]. Moreover, it has been reported that ErbB3 inactivation blocks proliferation of breast cancer cells in an ErbB2-independent manner [37]. As shown in Figure 5A, the ErbB3-3'-UTR carries a con- served $m i R-205$ binding site. Luciferase assays revealed that the reduction of luciferase activity by $m i R-205$ was dose-dependent (Figure 5B). To further confirm that miR-205-mediated reduction of the luciferase activity for Luc-ErbB3-3'-UTR vector is due to direct interaction between $m i R-205$ and its putative binding site, we mutated the $m i R-205$ binding site by site-directed mutagenesis, resulting in Luc-ErbB3-3'-UTR-d. As expected, $m i R$ 205-mediated suppression of the luciferase activity was completely abolished in Luc-ErbB3-3'-UTR-d (Figure $5 \mathrm{C}$ ), suggesting that the $m i R-205$ binding site is critical for $m i R-205$-mediated suppression. To further test the specificity of $m i R$-205-mediated suppression, we used anti-miR-205 oligo. Since 293 T cells express a low level of $m i R-205$, we first established stable clones overexpressing $m i R-205$ and then introduced anti-miR-205 into these stable transfectants. As shown in Figure 5D, anti$m i R-205$ enhanced reporter activity by over two-fold compared to scrambled oligo. 


\section{A}

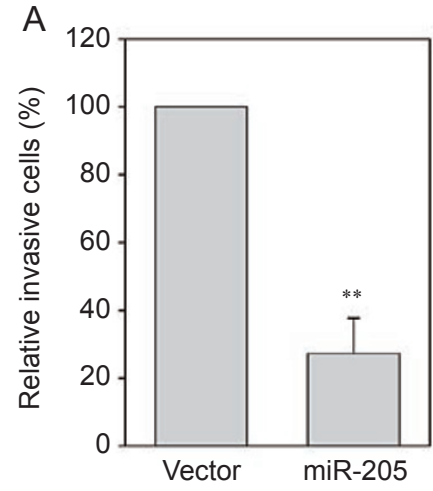

B
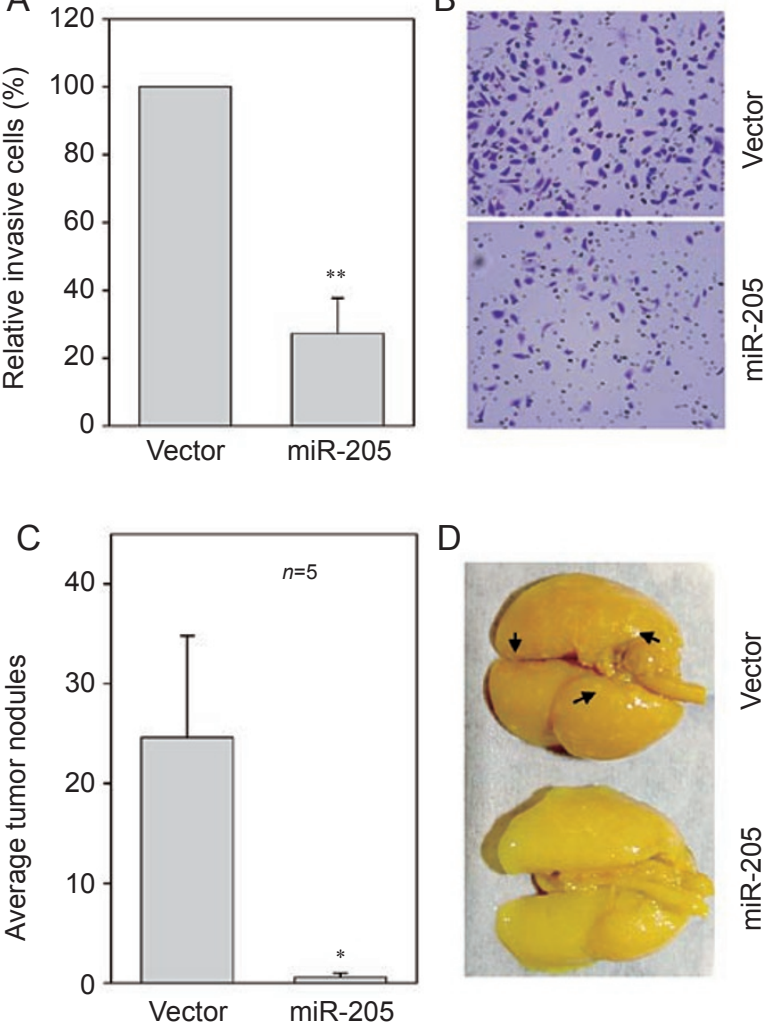

$\mathrm{D}$

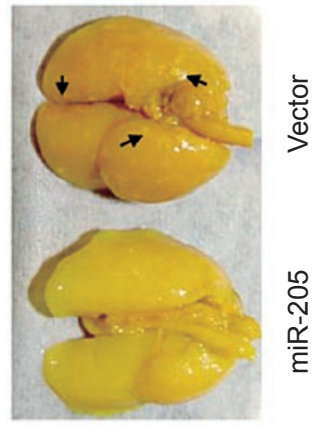

Figure 4 miR-205 inhibits cell invasion and metastasis. MDA-MB-231 cells were first infected with miR-205 or vector alone and then were tested for invasion ability in matrigel chambers or metastasis in nude mice as described in Materials and Methods. (A and B) In vitro cell invasion assays with representative fields of invaded cells. Values in (A) are means of three experiments \pm SE. $* * P<0.01$. (C) Effect of $m i R-205$ on tumor metastasis. $* P<0.05$. (D) Representative lungs harvested from the nude mice. Arrows indicate some of tumor nodules.

Likewise, VEGF-A is a key regulator of angiogenesis and is shown to play a key role particularly in tumor metastasis. As shown in Figure 5F, we similarly demonstrated that $m i R-205$ directly targets VEGF-A by interacting with the putative $m i R-205$ binding site in its 3'-UTR. Finally, anti-miR-205 also significantly enhanced the activity of Luc-VEGF-A-UTR (Figure 5G), suggesting the specificity of $m i R$-205-mediated suppression.

To determine whether miR-205 suppresses the expression of endogenous ErbB3, we performed western blot using transiently transfected MCF-7 cells. As shown in Figure 6A, miR-205 suppressed the ErbB3 level in MCF-7 cells significantly compared to vector control. Moreover, we detected even more dramatic reduction of VEGF-A in $m i R-205$-transfected MDA-MB-231 cells compared to vector control (Figure 6B). To better examine the suppression of ErbB3 at the cellular level, we performed immunofluorescence staining for transiently transfected MCF-7 cells. Since the miR-205 expression vector was tagged with GFP, the green cells represented miR-205-expressing cells. As shown in Figure $6 \mathrm{C}$, ectopic expression of $m i R-205$ clearly suppressed ErbB3 expression (arrows in middle panel). In contrast, the vector control had no effect on ErbB3 expression. Thus, these results further confirmed that $m i R-205$ can negatively regulate the expression of ErbB3.

\section{Discussion}

Growing evidence has indicated that miRNAs are frequently deregulated, and aberrant expression of a unique number of miRNAs (miRNA signature) is directly associated with a certain type of cancer. Our miRNA profiling suggests that $m i R-205$ is specifically downregulated in breast cancer, but not colon cancer. Moreover, $m i R$ 205 expression level is also decreased in breast cancer cell lines compared to the non-malignant breast epithelial cell line MCF-10A, suggesting $m i R-205$ as a potential tumor suppressor in breast cancer. In support of this notion, we demonstrate that ectopic expression of $m i R$ 205 suppresses proliferation, colonogenic survival and anchorage-independent growth of MCF-7 cells, and invasiveness in MDA-MB-231 cells. Therefore, miR-205 modulates not only breast tumor cell growth, but also cell invasion and metastasis.

While miR-205 is downregulated in breast cancer, it has been shown to be upregulated in various types of cancers, including lung cancer, bladder cancer, ovarian cancer and head and neck cancer cell lines [38-42]. In colon cancer, we detected no significant difference in miR-205 expression between tumor and the matched normal tissue. These findings along with this study may imply that $m i R-205$ could play a dual role in tumorigenicity, depending on tissue type and specific targets. In fact, such a dual nature of miRNAs in tumorigenicity has been previously reported. For instance, miR-155 is considered as an oncogene in many cancers because of its upregulation in these cancers, but the expression of miR-155 is significantly decreased in endocrine pancreatic tumors [43]. Studies on the miR-17-92 cluster have revealed that this miRNA cluster has tumor suppressive function by inhibiting E2F1 expression in human B-cell line, but the same miRNA cluster is overexpressed and acts as an oncogene by blocking apoptosis in B-cell lymphomas $[44,45]$. By using LNA-based microarrays and Northern blot analyses to measure miRNA levels in a collection of breast epithelial cell lines, Sempere et al. [46] demonstrated that $m i R-205$ was consistently downregulated in 
A 272

294

ErbB3 3'-UTR 5' -ggCACTCCTGGAGATATGAAGGA- 3'

miR-205 3'-gucUGAGG-CCACCUUACUUCCU- 5'
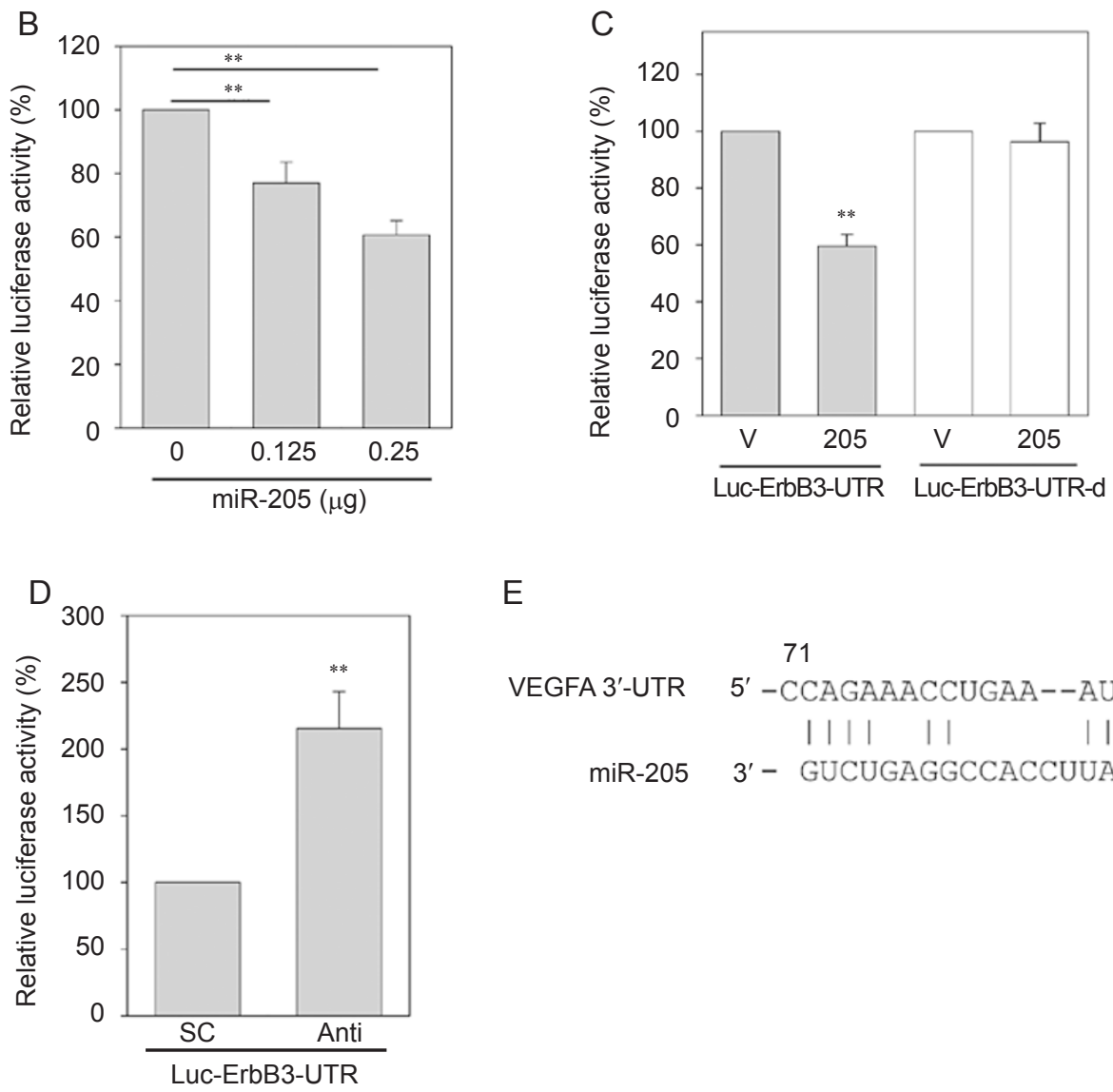

$\mathrm{E}$
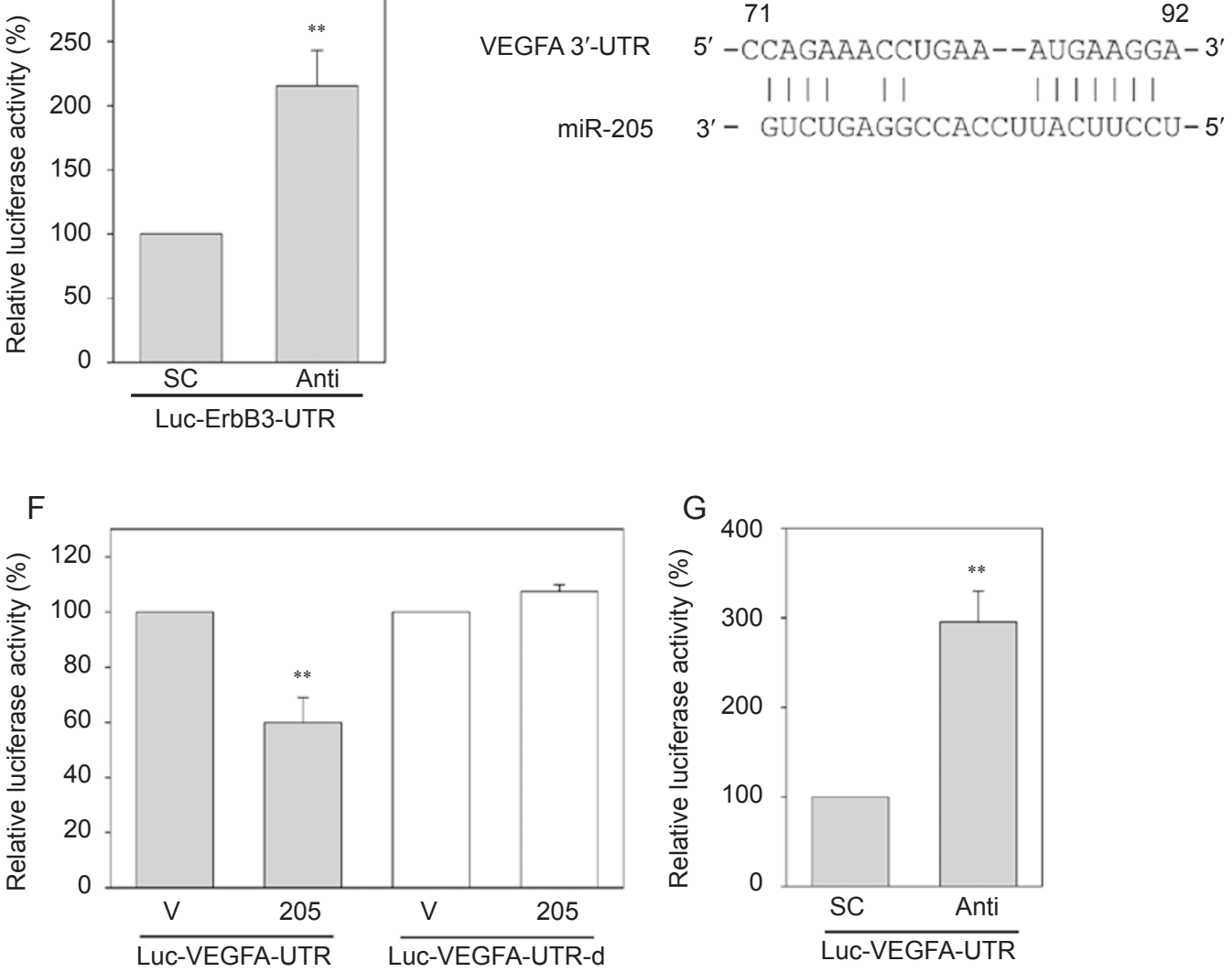

Figure 5 Identification of ErbB3 and VEGF-A as direct targets for miR-205. (A) Alignment of miR-205 with ErbB3 at the 3'UTR. (B) Suppression of the luciferase activity by miR-205 in a dose-dependent manner. (C) While miR-205 suppresses the luciferase activity for Luc-ErbB3-UTR, it has no effect on Luc-ErbB3-UTR-d. (D) Anti-miR-205 increases the luciferase activity of Luc-ErbB3-UTR in 293T cells that overexpress miR-205. (E) Alignment of miR-205 with VEGF-A at the 3'-UTR. (F) Effect of miR-205 on the luciferase activity of Luc-VEGF-A-UTR and Luc-VEGF-A-UTR-d. (G) Anti-miR-205 increases the luciferase activity of Luc-VEGF-A-UTR in 293T cells that overexpress miR-205. Values in (B, C, D, F and G) are means of three experiments \pm SE. ${ }^{* *} P<0.01$. SC, scrambled oligo; Anti, anti-miR-205. 


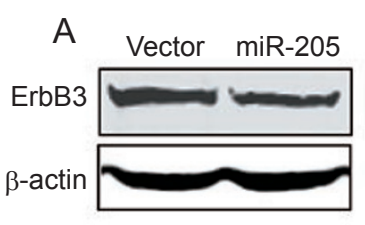

C
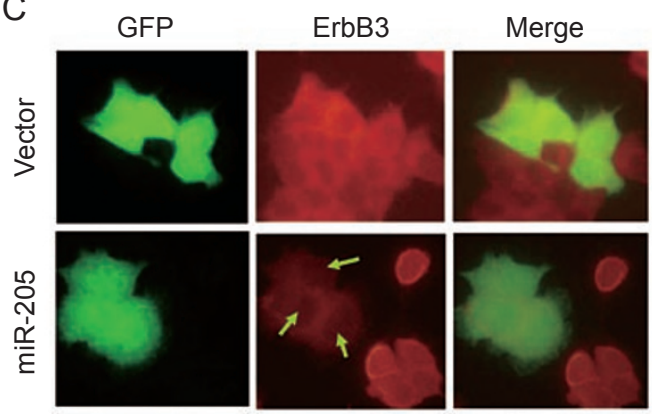

Figure 6 Suppression of the endogenous ErbB3 and VEGF-A by miR-205. (A and $\mathbf{B}$ ) Western blot revealing reduced level of ErbB3 in miR-205-transfected MCF-7 cells and reduced VEGF-A level in miR-205 MDA-MB-231 cells. (C) Detection of ErbB3 in MCF-7 cells by immunofluorescence staining. Note that the ErbB3 signal was very weak in the miR-205-transfected cells in the right panel.

tumorigenic cell lines, which is in good agreement with our finding. Given the opposite expression levels of $m i R$ 205 in different cancers, and the inhibitory effects of $m i R-205$ on proliferation, anchorage-independent growth and invasion ability of breast cancer cell lines, we suggest that $m i R-205$ is a breast cancer-specific tumor suppressor.

ErbB3 is a member of ErbB tyrosine kinase receptor family, which is frequently overexpressed in breast cancer. It is well known that there is a correlation between ErbB3 expression levels and tumorigenesis in breast cancer $[47,48]$. Because ErbB3 has an impaired tyrosine kinase activity, heterodimerization is required for ErbB3 activation and downstream signal transduction [36, 49]. By heterodimerizing with other family members, especially ErbB2, ErbB3 contributes to ErbB2-mediated proliferation and anti-cancer drug resistance of breast cancer cells $[37,50]$. Co-expression of ErbB2/ErbB3 heterodimer is a poor prognostic indicator. Therefore, identification of ErbB3 as a direct target for miR-205 may imply that $m i R-205$ is a novel target for breast cancer therapy.

Apparently, ErbB3 can also be targeted by other miRNAs than $m i R-205$. For example, a recent report indicates that both $m i R-125 a$ and $m i R-125 b$ can suppress ErbB3 expression by interacting with the same $m i R-125$ binding site located in the ErbB3 3'-UTR [51], suggesting that multiple miRNAs may have an additive or syner- getic effect on regulation of gene expression. In addition, lung metastasis can be enhanced by increasing ErbB3dependent signaling in orthotopic injection models of breast cancer [52]. On the other hand, since a single miRNA can have multiple targets, it is very likely that $m i R-205$ may also regulate other genes simultaneously to inhibit breast tumor growth. For example, miR-205 also targets VEGF-A, an important invasion and metastasis factor. Although VEGF-A is well known for its role in angiogenesis and tumor metastasis, the reduced level of VEGF-A could also contribute to the observed suppression of cell invasion in vitro (Figure 4), because a previous report indicates that VEGF-A is able to enhance cell invasion in vitro [53]. In addition to these two targets we identified in this study, $m i R-205$ may target other targets yet to be identified. Therefore, the observed miR-205mediated inhibition of breast tumor growth and metastasis is likely due to simultaneous targeting of multiple targets.

\section{Materials and Methods}

\section{Cell culture}

All cell lines used in this study were obtained from the American Type Culture Collection (ATCC) (Manassas, VA, USA). MCF-7, MDA-MB-231, MDA-MB-453 and MDA-MB-468 cells were grown in RPMI 1640 (Cambrex, Walkersville, MD, USA) supplemented with $10 \%$ fetal bovine serum (FBS) (Sigma-Aldrich), MDA-MB-435 cells were grown in Dulbecco's modified Eagle's medium (Cambrex) supplemented with $10 \%$ FBS plus $0.01 \mathrm{mg} / \mathrm{ml}$ insulin. MCF-10A cells were grown in Mammary Epithelial Basal Medium (Cambrex). 293T cells were grown in Dulbecco's modified Eagle's medium (Cambrex) supplemented with 10\% FBS. All media were supplemented with $2 \mathrm{mM}$ glutamine, 100 units of penicillin/ml, and $100 \mu \mathrm{g}$ of streptomycin/ml (Cambrex). Cells were incubated at $37{ }^{\circ} \mathrm{C}$ in a humidified chamber supplemented with $5 \% \mathrm{CO}_{2}$.

\section{Constructs}

To generate $m i R-205$ expression vector, a $\sim 600 \mathrm{bp}$ fragment carrying pre-miR-205 was amplified from MCF-10A genomic DNA by the high fidelity polymerase Phusion enzyme (New England Biolabs, Ipswich, MA, USA) using PCR primers miR-2055.1, 5'-GAATTC CTT ATC TGG GTG GCT GTT TTG and miR205-3.1, 5'-GGTACC GCG GTG CTT TTT CCA ATC TGC. The amplified fragment was first cloned into pCR8 (Invitrogen) and then subcloned into a modified $\mathrm{pCMV}$ vector carrying hygromycin resistance gene. To construct $m i R-205$ lentiviral expression vector, the same $m i R-205$ insert was released from pCMV vector and then subcloned into pCDH-CMV-MCS-EF1-copGFP (System Biosciences, Mountain View, CA, USA).

To generate a luciferase reporter carrying ErbB3-3'-UTR with a putative $m i R$-205 binding site, we amplified a 638-bp ErbB3 3'UTR region from MCF-7 cDNA using the following PCR primers: ErbB3-UTR-5.1, 5'-CTC CTG CTC CCT GTG GCA C and ErbB3-UTR-3.1, 5'-CCC GAC TTC CCT TTG TGT AAA ATG. 
The amplified fragment was first cloned into pCR8 vector and subsequently cloned into a modified pGL3-control vector [54] at the EcoR1 site. To delete the putative miR-205 binding site, we adopted two-step PCR methods as described previously [54]. Additional primers used were ErbB3-3'UTR-5.2, 5'-CAT TCC TCA GCT TCT TCA CAT TAC TCT CCA TAT CCC TTC C, and ErbB3-3'UTR-3.2, 5'- GGA AGG GAT ATG GAG AGT AAT GTG AAG AAG CTG AGG AAT G.

The same procedure was used to clone Luc-VEGF-A-UTR and Luc-VEGF-A-UTR-d, where the putative $m i R-205$ binding site was deleted. PCR primers were VFGF-A-UTR-5.1, 5'-TTT CGG GAA CCA GAT CTC TC; VEGF-A-UTR-3.1, 5'-GCGGCCGCT CTT CCC TGT CAG GAT CTG; VEGF-A-UTR-Del-5.1, 5'-CCA TCG ACA GAA CAG TCC TTA AGA GGA GAC TCT GCG CAG AG; VEGF-A-UTR-Del-3.1, 5'-CTC TGC GCA GAG TCT CCT CTT AAG GAC TGT TCT GTC GAT GG. All the PCR products were verified by DNA sequencing.

\section{Detection of miR-205 by real-time RT-PCR}

To detect mature $m i R-205$ expression in patient specimens, we used Trizol reagent to isolate total RNA, which was then amplified by TaqMan stem-loop RT-PCR method [30]. TaqMan microRNA assays used the Human Panel-Early Access Kit (ABI, Forest City, CA, USA), which includes 157 human miRNAs as well as 3 negative controls. We also used individual miR-205-specific primer sets and TaqMan probe from ABI to detect miR-205 expression in patient specimens and cell lines.

\section{Generation of miR-205 stable clones}

To establish miR-205 stable clones, MCF-7 cells were transfected with vector control or $m i R-205$ expression vector and were then selected in the presence of hygromycin at $200 \mu \mathrm{g} / \mathrm{ml} \mathrm{con-}$ centration. Individual colonies were expanded and expression of the exogenous miR-205 over the endogenous counterpart (vector alone) was determined by real-time RT-PCR.

\section{Cell proliferation assay}

Either transiently transfected cells or stable clones were seeded into 96-well plates at 1000 cells/well. Relative cell growth rate was determined by MTT assay as described previously [54].

\section{Clonogenic survival and anchorage-independent growth}

To determine the clonogenic survival of $m i R-205$ stable clones, cells from either vector control or $m i R-205$ were seeded on sixwell plates at 500 cells/well. At 10 days after seeding, colonies were fixed and stained with $0.1 \%$ crystal violet. Anchorage-independent growth of $m i R-205$ stable transfectants was tested according to a published method [55]. Briefly, $1 \mathrm{ml}$ culture medium with $0.4 \%$ agar was first plated into each well of a 12 -well plate. After the bottom agar solidified, each well received another $1 \mathrm{ml} 0.3 \%$ agar in culture medium carrying 5000 cells. Colonies were stained and counted after 2 weeks of incubation.

\section{Luciferase assay}

Luciferase reporters were transfected into $293 \mathrm{~T}$ cells along with either vector control or miR-205 expression vector by using the calcium phosphate method as described previously [56]. Cells were harvested $48 \mathrm{~h}$ after transfection for luciferase assay using a luciferase assay kit (Promega) according to the manufacturer's protocol. $\beta$-Galactosidase was used for normalization of transfection efficiency as described previously [54].

\section{Western blot}

Total protein was extracted from transient transfected MCF-7 cells or $m i R-205$ stable clones by using cell lysis buffer $(150 \mathrm{mM}$ $\mathrm{NaCl}, 10 \mathrm{mM}$ Tris, $\mathrm{pH} 7.2,0.1 \%$ SDS, $1.0 \%$ Triton $\mathrm{X}-100,1 \%$ Deoxycholate, $5 \mathrm{mM}$ EDTA). Protein concentration was measured with the Bio-Rad protein assay kit. The membrane was first probed with ErbB3 antibody (Cell Signaling Technology, Danvers, MA, USA), followed by secondary antibodies labeled with IRDye 800 (Rockland Immunochemicals, Gilbertsville, PA, USA). Signals were visualized using the Odyssey infrared imaging system (Li-Cor Biosciences, Lincoln, NE, USA).

\section{Immunofluorescence microscopy}

To detect the effect of $m i R-205$ on ErbB3 expression at the cellular level, MCF-7 cells were transfected with either miR-205/ pCDH-CMV-MCS-EF1-copGFP or control vector, pCDH-CMVMCS-EF1-copGFP. Immunodetection was performed as described previously [57]. In brief, transfected cells were seeded on cover slides $24 \mathrm{~h}$ after transfection, and further incubated for $96 \mathrm{~h}$ before fixing with $3 \%$ paraformaldehyde. After incubation with primary ErbB3 antibody, the second antibody conjugated with Alexa Fluor $560 \mathrm{~nm}$ (Invitrogen) was used to reveal ErbB3 signals. Images were taken under a fluorescent microscope (Olympus, Center Valley, PA, USA).

\section{Invasion assay}

Effect of $m i R-205$ on the invasion ability of MDA-MB-231 cells was determined using matrigel invasion chambers (BD Biosciences). Cells infected with either $m i R-205$ expression or control lentiviral vectors were seeded into inserts at $2 \times 10^{4}$ per insert in serum-free medium and then transferred to wells filled with the culture medium containing $10 \%$ FBS as a chemoattractant. After $24 \mathrm{~h}$ of incubation, non-invading cells on the top of the membrane were removed by scraping. Invaded cells on the bottom of the membrane were fixed, followed by staining with $0.05 \%$ crystal violet. The number of invaded cells on the membrane was then counted under a microscope.

\section{Tumor metastasis in vivo}

Animal studies were conducted to determine the effect of miR205 on breast tumor metastasis, in accordance with the NIH Animal Use Guidelines and a protocol approved by the SIU Animal Care Committee. The procedure involving the metastatic breast cancer cell line MDA-MB-231 cells has been described previously [58].

\section{Patient specimens}

Total RNA was isolated using Trizol reagent from freshly frozen specimens of matched breast tumors from the same patient, which were obtained from the Cooperative Human Tissue Network (CHTN) Midwestern Division (Columbus, OH, USA). The isolated RNA was directly used for TaqMan real-time PCR to detect expression of miRNAs.

\section{Acknowledgments}


This study was supported by grants to Y Mo from the National Cancer Institute, USA (CA102630) and from the Department of Defense (BC052294).

\section{References}

1 Bartel DP. MicroRNAs: genomics, biogenesis, mechanism, and function. Cell 2004; 116:281-297.

2 Mallory AC, Vaucheret H. Functions of microRNAs and related small RNAs in plants. Nat Genet 2006; 38 Suppl:S31-S36.

3 Jing Q, Huang S, Guth S, et al. Involvement of microRNA in AU-rich element-mediated mRNA instability. Cell 2005; 120:623-634.

4 Bracht J, Hunter S, Eachus R, Weeks P, Pasquinelli AE. Transsplicing and polyadenylation of let-7 microRNA primary transcripts. RNA 2004; 10:1586-1594.

5 Cai X, Hagedorn CH, Cullen BR. Human microRNAs are processed from capped, polyadenylated transcripts that can also function as mRNAs. RNA 2004; 10:1957-1966.

6 Lee Y, Kim M, Han J, et al. MicroRNA genes are transcribed by RNA polymerase II. EMBO J 2004; 23:4051-4060.

7 Han J, Lee Y, Yeom KH, et al. The Drosha-DGCR8 complex in primary microRNA processing. Genes Dev 2004; 18:30163027.

8 Yi R, Qin Y, Macara IG, Cullen BR. Exportin-5 mediates the nuclear export of pre-microRNAs and short hairpin RNAs. Genes Dev 2003; 17:3011-3016.

9 Lewis BP, Shih IH, Jones-Rhoades MW, Bartel DP, Burge CB. Prediction of mammalian microRNA targets. Cell 2003; 115:787-798.

10 Didiano D, Hobert O. Perfect seed pairing is not a generally reliable predictor for miRNA-target interactions. Nat Struct Mol Biol 2006; 13:849-851.

11 Grimson A, Farh KK, Johnston WK, et al. MicroRNA targeting specificity in mammals: determinants beyond seed pairing. Mol Cell 2007; 27:91-105.

12 Griffiths-Jones S, Grocock RJ, van Dongen S, Bateman A, Enright AJ. miRBase: microRNA sequences, targets and gene nomenclature. Nucleic Acids Res 2006; 34:D140-D144.

13 Berezikov E, Guryev V, van de Belt J, et al. Phylogenetic shadowing and computational identification of human microRNA genes. Cell 2005; 120:21-24.

14 He L, Hannon GJ. MicroRNAs: small RNAs with a big role in gene regulation. Nat Rev Genet 2004; 5:522-531.

15 Lecellier $\mathrm{CH}$, Dunoyer P, Arar K, et al. A cellular microRNA mediates antiviral defense in human cells. Science 2005; 308:557-560.

16 Sullivan CS, Grundhoff AT, Tevethia S, Pipas JM, Ganem D. SV40-encoded microRNAs regulate viral gene expression and reduce susceptibility to cytotoxic T cells. Nature 2005; 435:682-686.

17 Lewis BP, Burge CB, Bartel DP. Conserved seed pairing, often flanked by adenosines, indicates that thousands of human genes are microRNA targets. Cell 2005; 120:15-20.

18 Calin GA, Dumitru CD, Shimizu M, et al. Frequent deletions and down-regulation of micro- RNA genes miR15 and miR16 at 13 q14 in chronic lymphocytic leukemia. Proc Natl Acad Sci USA 2002; 99:15524-15529.

19 Calin GA, Croce CM. MicroRNA signatures in human can- cers. Nat Rev Cancer 2006; 6:857-866.

20 Porkka KP, Pfeiffer MJ, Waltering KK, et al. MicroRNA expression profiling in prostate cancer. Cancer Res 2007; 67:6130-6135.

21 Iorio MV, Ferracin M, Liu CG, et al. MicroRNA gene expression deregulation in human breast cancer. Cancer Res 2005; 65:7065-7070.

22 Calin GA, Sevignani C, Dumitru CD, et al. Human microRNA genes are frequently located at fragile sites and genomic regions involved in cancers. Proc Natl Acad Sci USA 2004; 101:2999-3004.

23 Lim LP, Glasner ME, Yekta S, Burge CB, Bartel DP. Vertebrate microRNA genes. Science 2003; 299:1540.

24 Wienholds E, Kloosterman WP, Miska E, et al. MicroRNA expression in zebrafish embryonic development. Science 2005; 309:310-311.

25 Landgraf P, Rusu M, Sheridan R, et al. A mammalian microRNA expression atlas based on small RNA library sequencing. Cell 2007; 129:1401-1414.

26 Ason B, Darnell DK, Wittbrodt B, et al. Differences in vertebrate microRNA expression. Proc Natl Acad Sci USA 2006; 103:14385-14389.

27 Shingara J, Keiger K, Shelton J, et al. An optimized isolation and labeling platform for accurate microRNA expression profiling. $R N A$ 2005; 11:1461-1470.

28 Chen CZ. MicroRNAs as oncogenes and tumor suppressors. $N$ Engl J Med 2005; 353:1768-1771.

29 Si ML, Zhu S, Wu H, et al. miR-21-mediated tumor growth. Oncogene 2007; 26:2799-2803.

30 Chen C, Ridzon DA, Broomer AJ, et al. Real-time quantification of microRNAs by stem-loop RT-PCR. Nucleic Acids Res 2005; 33:e179.

31 Colburn NH, Bruegge WF, Bates JR, et al. Correlation of anchorage-independent growth with tumorigenicity of chemically transformed mouse epidermal cells. Cancer Res 1978; 38:624-634.

32 Gregory PA, Bert AG, Paterson EL, et al. The miR-200 family and miR-205 regulate epithelial to mesenchymal transition by targeting ZEB1 and SIP1. Nat Cell Biol 2008; 10:593-601.

33 Fidler IJ. The pathogenesis of cancer metastasis: the 'seed and soil' hypothesis revisited. Nat Rev Cancer 2003; 3:453-458.

34 Krek A, Grun D, Poy MN, et al. Combinatorial microRNA target predictions. Nat Genet 2005; 37:495-500.

35 John B, Enright AJ, Aravin A, et al. Human MicroRNA targets. PLoS Biol 2004; 2:e363.

36 Naidu R, Yadav M, Nair S, Kutty MK. Expression of c-erbB3 protein in primary breast carcinomas. Br J Cancer 1998; 78:1385-1390.

37 Holbro T, Beerli RR, Maurer F, et al. The ErbB2/ErbB3 heterodimer functions as an oncogenic unit: ErbB2 requires ErbB3 to drive breast tumor cell proliferation. Proc Natl Acad Sci USA 2003; 100:8933-8938.

38 Volinia S, Calin GA, Liu CG, et al. A microRNA expression signature of human solid tumors defines cancer gene targets. Proc Natl Acad Sci USA 2006; 103:2257-2261.

39 Gottardo F, Liu CG, Ferracin M, et al. Micro-RNA profiling in kidney and bladder cancers. Urol Oncol 2007; 25:387-392.

40 Tran N, McLean T, Zhang X, et al. MicroRNA expression profiles in head and neck cancer cell lines. Biochem Biophys Res 
Commun 2007; 358:12-17.

41 Iorio MV, Visone R, Di Leva G, et al. MicroRNA signatures in human ovarian cancer. Cancer Res 2007; 67:8699-8707.

42 Yanaihara N, Caplen N, Bowman E, et al. Unique microRNA molecular profiles in lung cancer diagnosis and prognosis. Cancer Cell 2006; 9:189-198.

43 Roldo C, Missiaglia E, Hagan JP, et al. MicroRNA expression abnormalities in pancreatic endocrine and acinar tumors are associated with distinctive pathologic features and clinical behavior. J Clin Oncol 2006; 24:4677-4684.

44 O'Donnell KA, Wentzel EA, Zeller KI, Dang CV, Mendell JT. c-Myc-regulated microRNAs modulate E2F1 expression. $\mathrm{Na}$ ture 2005; 435:839-843.

45 He L, Thomson JM, Hemann MT, et al. A microRNA polycistron as a potential human oncogene. Nature $2005 ;$ 435:828-833.

46 Sempere LF, Christensen M, Silahtaroglu A, et al. Altered MicroRNA expression confined to specific epithelial cell subpopulations in breast cancer. Cancer Res 2007; 67:11612-11620.

47 Guy PM, Platko JV, Cantley LC, Cerione RA, Carraway KL. 3rd Insect cell-expressed p180erbB3 possesses an impaired tyrosine kinase activity. Proc Natl Acad Sci USA 1994; 91:8132-8136.

48 Kim HH, Vijapurkar U, Hellyer NJ, Bravo D, Koland JG. Signal transduction by epidermal growth factor and heregulin via the kinase-deficient ErbB3 protein. Biochem J 1998; 334 (Part 1):189-195.

49 Lemoine NR, Barnes DM, Hollywood DP, et al. Expression of the ERBB3 gene product in breast cancer. Br J Cancer 1992; 66:1116-1121.
50 Liu B, Ordonez-Ercan D, Fan Z, et al. Downregulation of erbB3 abrogates erbB2-mediated tamoxifen resistance in breast cancer cells. Int J Cancer 2007; 120:1874-1882.

51 Scott GK, Goga A, Bhaumik D, et al. Coordinate suppression of ERBB2 and ERBB3 by enforced expression of micro-RNA miR-125a or miR-125b. J Biol Chem 2007; 282:1479-1486.

52 Xue C, Liang F, Mahmood R, et al. ErbB3-dependent motility and intravasation in breast cancer metastasis. Cancer Res 2006; 66:1418-1426.

53 Bachelder RE, Wendt MA, Mercurio AM. Vascular endothelial growth factor promotes breast carcinoma invasion in an autocrine manner by regulating the chemokine receptor CXCR4. Cancer Res 2002; 62:7203-7206.

54 Zhu S, Si ML, Wu H, Mo YY. MicroRNA-21 targets the tumor suppressor gene tropomyosin 1 (TPM1). J Biol Chem 2007; 282:14328-14336.

55 Finlay TH, Tamir S, Kadner SS, et al. alpha 1-Antitrypsin- and anchorage-independent growth of MCF-7 breast cancer cells. Endocrinology 1993; 133:996-1002.

56 Mo YY, Beck WT. Association of human DNA topoisomerase IIalpha with mitotic chromosomes in mammalian cells is independent of its catalytic activity. Exp Cell Res 1999; 252:50-62.

57 Wu F, Chiocca S, Beck WT, Mo YY. Gam1-associated alterations of drug responsiveness through activation of apoptosis. Mol Cancer Ther 2007; 6:1823-1830.

$58 \mathrm{Zhu} \mathrm{S}, \mathrm{Wu} \mathrm{H}, \mathrm{Wu}$ F, et al. MicroRNA-21 targets tumor suppressor genes in invasion and metastasis. Cell Res 2008; 18:350-359. 